\title{
Holographic optics and negative refractive index
}

\author{
Antonio Amariti, ${ }^{a}$ Davide Forcella, ${ }^{b}$ Alberto Mariotti ${ }^{c}$ and Giuseppe Policastro ${ }^{b}$ \\ ${ }^{a}$ Department of Physics, University of California, \\ San Diego La Jolla, CA 92093-0354, U.S.A. \\ ${ }^{b}$ Laboratoire de Physique Théorique de l'École Normale Supérieure and CNRS UMR 8549, \\ 24 Rue Lhomond, Paris 75005, France \\ ${ }^{c}$ Theoretische Natuurkunde, Vrije Universiteit Brussel and The International Solvay Institutes, \\ Pleinlaan 2, B-1050 Brussels, Belgium \\ E-mail: amariti@ucsd.edu, forcella@lpt.ens.fr, \\ alberto.mariotti@vub.ac.be, policast@lpt.ens.fr
}

ABSTRACT: In recent years a very exciting and intense activity has been devoted to the understanding and construction of materials that enjoy exotic optical properties, such as a negative refractive index. Motivated by these experimental and theoretical developments, we use the string-inspired idea of holography to study the electromagnetic response of a certain class of media: strongly coupled relativistic systems that admit a dual gravitational description. Our results indicate that this type of media generally have a negative refractive index. Moreover we observe that a negative refractive index could be a common feature of relativistic hydrodynamic systems at low frequencies.

KeYwords: Gauge-gravity correspondence, Electromagnetic Processes and Properties, Holography and quark-gluon plasmas 


\section{Contents}

1 Introduction $\quad 1$

2 Macroscopic EM and linear response theory 3

3 Negative refraction $\quad 5$

4 The holographic model $\quad 8$

$\begin{array}{lll}5 & \text { Numerics and plots } & 10\end{array}$

6 Conclusions and future directions $\quad 13$

$\begin{array}{ll}\text { A Wave propagation and dissipation } & 14\end{array}$

$\begin{array}{ll}\text { B Finding the correlators } & 16\end{array}$

\section{Introduction}

It is a well-known fact that there exists a close analogy between the free propagation of light in curved spacetime and the propagation of light inside a material in flat space. The geometry bends the light trajectories along null geodesics of the spacetime metric $g_{\mu \nu}$, while the electromagnetic properties of the medium deviate the trajectory of the light according to its electric and magnetic tensors: $\epsilon_{i j}, \mu_{i j}$. Indeed it is formally possible to rewrite the microscopic Maxwell equations in curved spacetime as macroscopic Maxwell equations in a continuos medium in flat spacetime (see for example [1]).

This old story takes a new turn with the recent discovery that one can engineer "real" materials with highly unusual and astonishing electromagnetic properties $[2,3]$. They are called metamaterials, since they are artificial materials engineered to provide properties which are not readily available in nature. They are typically constructed using periodic arrays of very small and closely spaced elements, playing the role of atoms in natural materials. One can engineer materials that force the light to follow almost any desired trajectory, allowing the construction of cloaking devices, perfect lenses, photonic black holes and so on. We cannot do justice to the literature here, we refer the reader to the reviews $[1,4-7]$.

One of the most interesting properties of some metamaterials is the negative refractive index. ${ }^{1}$ The theoretical possibility of this occurrence was first envisaged in 1968 by Veselago [8], although there are earlier suggestions due to Lamb, Schuster, and Mandelstam, see [9-11] and [12] for a historical account. In such a material the phase of a wave packet propagates in the opposite direction to the energy flux, which in turn leads to a number

\footnotetext{
${ }^{1}$ Materials with this property are also known as Left-Handed Materials.
} 
of surprising phenomena: many familiar laws of optics are modified, e.g. the Snell's law, the Doppler effect, the Cherenkov effect etc. Around 2000 the first samples of negative refractive materials (NRM) were built $[2,3,13]$ and since then they have attracted intense interest, due to their multiple technological applications.

It would be important to find new and general classes of physical systems that exhibit negative refraction, and for which it is possible to exactly compute some relevant physical quantities, at least at the linear response level. In this paper we observe that the negative refraction seems to be a generic prediction of relativistic hydrodynamics. More precisely, we show that if the correlator of the transverse current coupling to the EM field is dominated by a diffusive pole then there is a low-frequency region where the negative refraction occurs. The presence of the diffusive pole is a consequence of the hydrodynamic equations in the case of relativistic field theories at finite temperature and chemical potential.

We study in detail the refractive index for the specific example of a strongly coupled medium (eventually coupled to a dynamical electromagnetic field) that admits a gravity dual weakly coupled description. The computation of the transport coefficients and of the thermodynamical quantities is performed by using the string theory-inspired approach of holography. The holographic analysis goes well beyond the hydrodynamic regime, because the response functions are calculated at every frequency. Our results show that certain strongly coupled systems, coupled to a dynamical electromagnetic field, have negative refractive index in some specified range of frequencies. This confirms the hydrodynamic argument and introduces a new connection between gravity and optics, based on the idea of holography, realized in string theory by the AdS/CFT correspondence [14].

The AdS/CFT correspondence is a map between a quantum field theory in flat space, and a classical field theory in a completely different spacetime - a curved one, and with a larger number of dimensions. Often this is an Anti-de Sitter space or some deformation of it. The correspondence is holographic, since one can interpret the flat spacetime of the QFT as being the boundary of the curved spacetime. In this setup the geometric properties of the higher dimensional curved spacetime are translated in properties of matter in flat spacetime (see [15] for a review).

In the last decade, this holographic duality has been used with the aim to model several systems realized in the laboratories. The first example was the quark-gluon plasma produced in heavy-ion collisions [16-18]. This was followed by holographic realizations of many phenomena of condensed matter systems: superconductivity and superfluidity [19-23], Fermi gas at unitarity [24, 25], the quantum Hall effect [26-28], non-Fermi liquids [29-33], quantum phase transitions [34-38]. See [39-43] for reviews.

This paper is a first attempt towards an optics/geometry holographic duality, where string theory can provide a useful description for some unusual optical properties of electromagnetic media.

We believe that our results could be interesting both for the optics community, because they offer new theoretical examples and a "laboratory" for negative refractive index materials, in which the electromagnetic properties of the medium can be computed explicitly; and for the string theorists, because they provide a new exciting field of research in the AdS/CFT correspondence. 
We should mention at the outset a generic caveat of this string theory setup. The systems we are studying are not automatically coupled to a dynamical photon, so there is strictly speaking no propagation of light; nevertheless we speak of the refractive index. We can do it because, at the first order in the electromagnetic coupling, the optical properties of the medium are completely determined by the linear response to an external electromagnetic field, which can just be taken as a background, non-dynamical field. At the phenomenological level we can think of our system as a strongly interacting medium weakly coupled to a dynamical photon. The first part of the system is studied using the holography, while the coupling to the photon is taken into account perturbatively at the linearized level.

We should also notice that the systems we study are relativistic, homogeneous, isotropic, strongly-coupled media. We cannot pretend that our results can be directly applied to the real metamaterials that in particular are non-relativistic. It would be interesting to understand if our results can give experimentally testable predictions for systems that occur in nature (perhaps in neutron stars or other exotic situations). Moreover it could be interesting to study linear the EM response in non-homogeneous, non-isotropic or non-relativistic holographic setups.

The paper is organized as follows: in section 2 we recall the formalism of electromagnetic fields in a medium and the linear response theory that allows to derive the EM response tensors. In section 3 we discuss the conditions under which negative refraction of light occurs, and we give our main result: negative refraction is generic in a certain class of relativistic systems. In section 4 we describe the holographic setup and the techniques of the computation. In section 5 we give more details on the results, then we conclude with some additional comments and future directions of investigation. In the appendix we discuss some details regarding both the optics and the holographic setup.

\section{Macroscopic EM and linear response theory}

The analysis of the electrodynamics of continuos media is traditionally performed by introducing, in addition to the electric and magnetic field, $E$ and $B$, the macroscopic fields $D$ and $H$. They are not independent fields but contain the information about the response of the medium to the application of an external field. The general relation, at the linearized level, i.e. for weak external fields, has the form

$$
\begin{aligned}
& D_{i}(x, t)=\int \epsilon_{i j}\left(t-t^{\prime}\right) E_{j}\left(x, t^{\prime}\right) d t^{\prime}, \\
& B_{i}(x, t)=\int \mu_{i j}\left(t-t^{\prime}\right) H_{j}\left(x, t^{\prime}\right) d t^{\prime} .
\end{aligned}
$$

In this approach the properties of the medium are encoded in the (Fourier-transformed) response functions $\epsilon_{i j}(\omega), \mu_{i j}(\omega)$. They are often assumed to be frequency-dependent only. In an isotropic medium, moreover, they must reduce to scalar functions: $\epsilon_{i j}(\omega)=\epsilon(\omega) \delta_{i j}$, $\mu_{i j}(\omega)=\mu(\omega) \delta_{i j}$.

If the medium has spatial dispersion the response functions also depend on the wave vector $k$. In this case it is possible [44] to reduce the number of macroscopic fields to $D$, 
$E, B$, and use the single function $\epsilon_{i j}(\omega, k)$ to describe the linear response of the medium:

$$
D_{i}=\epsilon_{i j}(\omega, k) E_{j}
$$

For isotropic media the dielectric tensor $\epsilon_{i j}$ can be decomposed in its transverse and longitudinal part as

$$
\epsilon_{i j}=\epsilon_{T}(\omega, k)\left(\delta_{i j}-\frac{k_{i} k_{j}}{k^{2}}\right)+\epsilon_{L}(\omega, k) \frac{k_{i} k_{j}}{k^{2}}
$$

The dispersion relations are obtained by projecting the Maxwell equations on the transverse and longitudinal components. We have

$$
\epsilon_{T}(\omega, k)=\frac{k^{2}}{\omega^{2}}, \quad \epsilon_{L}(\omega, k)=0
$$

In experimental optics the most common description is not in term of $\epsilon_{T}$ and $\epsilon_{L}$ but it is in terms of $\epsilon$ and $\mu$. The two approaches can be related in the following way. At $k=0$ one has $\epsilon_{T}=\epsilon_{L}=\epsilon(\omega)$. If we expand the transverse dielectric permittivity as a series in $k$, we can write $[9-11]$

$$
\epsilon_{T}(\omega, k)=\epsilon(\omega)+\frac{k^{2}}{\omega^{2}}\left(1-\frac{1}{\mu(\omega)}\right)+\mathcal{O}\left(k^{4}\right) .
$$

From (2.4) and (2.5), keeping the terms up to $k^{2}$, we find that the transverse dispersion relation can be written as

$$
\frac{k^{2}}{\omega^{2}}=n^{2}(\omega)=\epsilon(\omega) \mu(\omega)
$$

where $n(\omega)$ represents the refractive index, and this is the same relation usually obtained in the $\epsilon-\mu$ approach. The two approaches are equivalent when the spatial dispersion is small, and one can neglect the higher-order terms in the expansion (2.5).

It is important to note that the $\mu(\omega)$ defined via the eq. (2.5) is not the same that appears in (2.1). It is instead an effective $\mu$ containing both electric and magnetic effects. The magnetic permeability, as commonly defined, can be obtained from the response functions as $[44]$

$$
1-\frac{1}{\mu(\omega)}=\omega^{2} \lim _{k \rightarrow 0} \frac{\epsilon_{T}(\omega, k)-\epsilon_{L}(\omega, k)}{k^{2}} .
$$

It is interesting to remark that the qualitative results of this paper do not change using this definition of $\mu$. However it is the effective $\mu$ that correctly reproduces the dispersion relation and hence the electromagnetic propagation and dissipation. ${ }^{2}$

The electric permittivity and the magnetic permeability can be obtained from linear response theory [45]. In the linear response theory the electromagnetic current $J_{i}$ is proportional to the vector potential $A_{j}, J_{i}=G_{i j} A_{j}$, where $G_{i j}$ is the retarded correlator of the currents in the medium:

$$
G_{i j}\left(x-x^{\prime}, t-t^{\prime}\right)=-i \theta\left(t-t^{\prime}\right)\left\langle\left[J_{i}(x, t), J_{j}\left(x^{\prime}, t^{\prime}\right)\right]\right\rangle .
$$

\footnotetext{
${ }^{2}$ Notice that the magnetic permeability $\mu$ can lose its significance at relatively small frequencies [44].
} 
We use for $G$ the same decomposition in transverse and longitudinal part used in eq. (2.3), and using the macroscopic Maxwell equations we obtain:

$$
\epsilon_{T}(\omega, k)=1-\frac{4 \pi}{\omega^{2}} q^{2} G_{T}(\omega, k) .
$$

where $q$ is the four-dimensional EM coupling. Expanding to second order in $k: G_{T}(\omega, k)=$ $G_{T}^{(0)}(\omega)+k^{2} G_{T}^{(2)}(\omega)$, we find the electric permittivity and effective magnetic permeability as

$$
\begin{aligned}
& \epsilon(\omega)=1-\frac{4 \pi}{\omega^{2}} q^{2} G_{T}^{(0)}(\omega) \\
& \mu(\omega)=\frac{1}{1+4 \pi q^{2} G_{T}^{(2)}(\omega)} \simeq 1-4 \pi q^{2} G_{T}^{(2)}(\omega)
\end{aligned}
$$

where in the second line we have expanded for weak EM coupling $q$. In summary, the response functions, determining the propagation of light in the medium, are given in terms of the retarded correlator of transverse currents. ${ }^{3}$

\section{$3 \quad$ Negative refraction}

As we just discussed, the propagation of electromagnetic waves in a medium is usually described in terms of the refractive index $n$ defined as $n^{2}=\epsilon \mu$. In the absence of dissipation $\epsilon$ and $\mu$ are real. In this case, there is wave propagation in the material only if $\epsilon \mu>0$. The definition of $n$ does not seem sensitive to the simultaneous change of sign of both $\epsilon$ and $\mu$, since the refractive index is defined as a quadratic equation. However, it was understood quite some time ago [8] that changing the sign of $\epsilon$ and $\mu$ corresponds to changing the branch in the square root, i.e. passing from $n=+\sqrt{\epsilon \mu}$, positive refractive index, to $n=-\sqrt{\epsilon \mu}$, negative refractive index.

The phase velocity of a wave is defined as $v_{p h}=1 / \operatorname{Re}(n)$, and the change of sign corresponds to the change of direction of the phase velocity. On the other hand the direction of the Poynting vector, and then of the energy flow, is not affected by this change of sign. We are in the exotic situation in which the energy flow and the phase velocity are opposite. In this case many physical laws change and the material has very special properties such as inverse Doppler and Cherenkov effect, inverse Snell's law, the medium could work as a perfect lens, etc.

However, the situation with constant and negative $\epsilon$ and $\mu$ is non physical [4]. One needs $\epsilon$ and $\mu$ to have a dependence on the frequency. In this case of frequency dispersion, from general principles we expect $\epsilon$ and $\mu$ to acquire an imaginary part, so that the medium is dissipative. In these media $n$ is itself a complex quantity. Its real part is the index of refraction while its imaginary part (usually referred as the extinction coefficient) takes into account the dissipation, and it is always positive in lossy materials. Differently from the

\footnotetext{
${ }^{3}$ Longitudinal waves can also propagate if there are solutions of the second equation of (2.4), and they could also in principle exhibit negative refraction, but in this paper we will only consider the transverse modes.
} 
case of real $\epsilon$ and $\mu$, here the refraction can be negative also if $\operatorname{Re}(\epsilon)$ and $\operatorname{Re}(\mu)$ are not simultaneously negative, because of the presence of the imaginary parts.

For the dissipative case, many different equivalent conditions for negative refraction have been worked out $[46,47]$. In [47] (as we review in appendix A) it was shown that the energy flow and the phase velocity are opposite if and only if the index

$$
n_{D L}(\omega)=|\epsilon(\omega)| \operatorname{Re}(\mu(\omega))+|\mu(\omega)| \operatorname{Re}(\epsilon(\omega))
$$

is negative. In the opposite case we have normal wave propagation. The condition $n_{D L}<0$ is equivalent to require that the refractive index is negative. In this paper we will use $n_{D L}$ to check if the medium has positive or negative refraction.

It is natural to wonder for which kind of media $n_{D L}<0$. In the following we study the behavior of $n_{D L}$ in systems that admit an hydrodynamic description for low frequencies and long wave vector, and argue that there is a simple condition that implies $n_{D L}<0$ in these systems. The basic aspects of hydrodynamics and correlation functions can be found in [48]. We assume the existence of a current $J$ such that its transverse part $J_{T}$ has a diffusive behavior: $\left(\partial_{t}-\mathcal{D} \nabla^{2}\right) J_{T}=0$, where $\mathcal{D}$ is the diffusion coefficient. This implies that the retarded correlator of $J_{T}$ has a diffusive pole, and it has the form

$$
G_{T}(\omega, k)=\frac{i \mathcal{B} \omega}{i \omega-\mathcal{D} k^{2}}
$$

with $\mathcal{B}$ a real number, up to contact terms and terms that are subdominant in the hydrodynamic limit (i.e. they are of higher order in $\omega, k^{2}$ ). From generic properties of the Green functions we know that: $\mathcal{D}>0$ (causality implies that the poles can only lie in the lower complex half-plane) and $\mathcal{B}>0\left(\operatorname{Im} G_{T}<0\right)$. If $J_{T}$ couples to the EM field with coupling $q$, the electric permittivity and magnetic permeability are

$$
\epsilon(\omega)=1-\frac{4 \mathcal{B} \pi q^{2}}{\omega^{2}}, \quad \mu(\omega)=1+\frac{4 i \mathcal{B D} \pi q^{2}}{\omega}
$$

From these expressions it is easy to show that $n_{D L}<0$ if $\omega^{2}<4 \pi q^{2} \mathcal{B}$ : every system that couples to the EM field with a transverse conserved current with retarded correlator as in (3.2) shows negative refraction at low enough frequencies.

The analysis of the hydrodynamic equations for a relativistic system at finite temperature and chemical potential $[48,49]$ shows that the transverse current indeed satisfies a diffusion equation; it is also possible to relate the constants $\mathcal{B}$ and $\mathcal{D}$ to the values of the transport coefficients and thermodynamical quantities:

$$
\mathcal{B}=\frac{\rho^{2}}{\varepsilon+P}, \quad \mathcal{D}=\frac{\eta}{\varepsilon+P}
$$

$\varepsilon$ is the energy density, $\rho$ the charge density, $P$ the pressure and $\eta$ the shear viscosity. Even though it is a simple prediction of hydrodynamics, ${ }^{4}$ this observation is new, to our knowledge. It would be interesting to perform a detailed analysis of the EM linear response

\footnotetext{
${ }^{4}$ We are grateful to Sean Hartnoll for suggesting us to investigate the generic structure of the correlators dictated by relativistic hydrodynamics.
} 


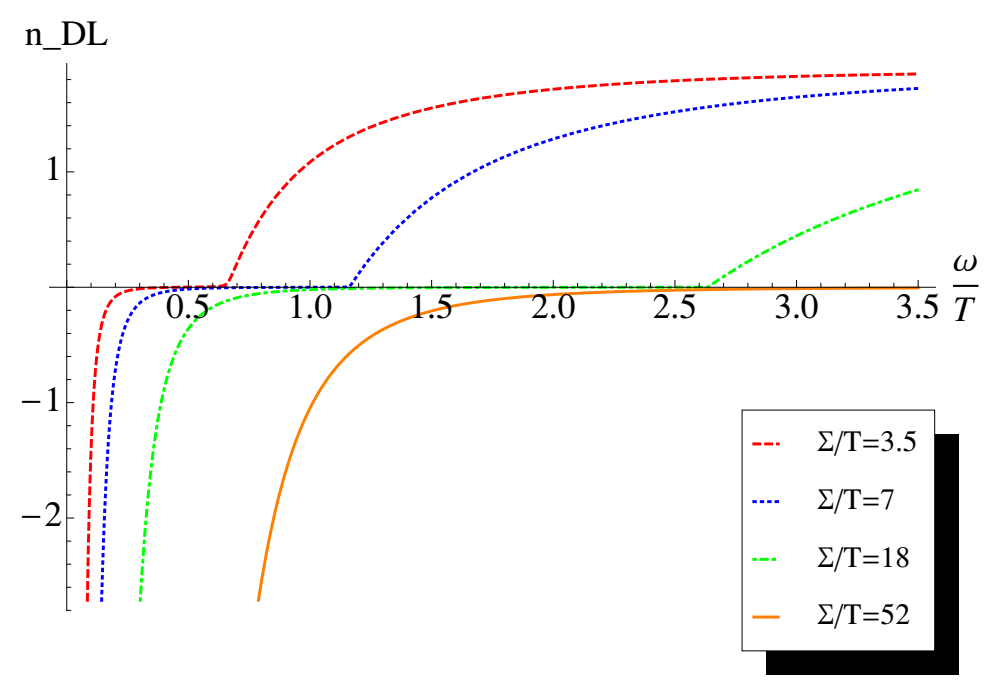

Figure 1. $n_{D L}$ for different values of $\Sigma / T$ and $q=0.05$.

functions for a generic relativistic hydrodynamic system at finite temperature and chemical potential, and give a general claim about negative refraction at low frequencies.

From now on we concentrate on a specific class of systems: strongly coupled plasmas at finite temperature and chemical potential, eventually coupled to a dynamical photon, that admit a dual geometric description. They are very interesting for two main reasons: it is possible to explicit compute the transport coefficients and the thermodynamical quantities, and it is possible to study the transverse current-current response function for every frequency, going well beyond the hydrodynamic regime. Even if they are strongly coupled systems we can perform the calculations by using the holographic approach. We find a range of frequencies where the refractive index is negative, for a generic class of these materials. In particular the transverse $G_{T}(\omega, k)$ behaves as (3.2) and the constants are given in term of the coefficients in (3.4), for low frequencies.

In figure 1 we show the behavior of the index $n_{D L}$ for different values of the ratio $\Sigma / T$, where $\Sigma$ is the chemical potential, and $T$ the temperature.

We observe that, as expected, at low frequencies there is negative refraction, whereas for high frequencies there is positive refraction. In the limit of zero chemical potential the index $n_{D L}$ is always positive and there is no negative refraction. As the charge is switched on, a region of negative refraction appears. This region becomes larger as the charge increase.

Before giving some details of the system and the computations, it is important to discuss the caveat of the dynamical photon that we already outlined in the introduction. It is well-known that generically in the holographic correspondence a local U(1) symmetry in the bulk corresponds to a global U(1) symmetry in the boundary. As a consequence there is no dynamical photon in the dual field theory described by the five dimensional geometry. As a natural way out we could consider our system as a strongly interacting medium weakly 
coupled to the gauge field associated to the global current we are considering. We use the holographic correspondence to describe only the medium and we add by hand, in a second time, the corrections induced by the presence of a dynamical photon. We would like to argue that this is a sensible procedure. The propagator for a dynamical photon in a given medium is [50]:

$$
\frac{4 \pi}{\omega^{2} \epsilon_{T}^{q}(\omega, k)-k^{2}}
$$

where $q$ is the coupling between the current and the photon: $q J_{\mu} A^{\mu}$, and $\epsilon_{T}^{q}(\omega, k)=1-$ $\frac{4 \pi}{\omega^{2}} q^{2} G_{T}^{q}(\omega, k) . G_{T}^{q}(\omega, k)$ is the full retarded current-current correlator. It takes into account the interactions among the constituents of the medium and the interactions between the medium and the dynamical photon. If the medium and the photon are weakly coupled the current-current correlator has a natural expansion in power of the coupling constant $q$ : $G_{T}^{q}(\omega, k)=G_{T}(\omega, k)+q^{2} G_{T}^{(2)}(\omega, k)+\ldots$ In this paper we are able to compute only the first term of this series: $G_{T}(\omega, k)$. It is the leading term in perturbative expansion of (3.5) and we could be confident that we are considering the leading effects for the propagation of a dynamical photon weakly coupled to the strongly coupled medium. The same philosophy has been applied to the computation of the photo-production rate from the quark-gluon plasma in [51].

\section{The holographic model}

In this section we introduce our model and we explain in which sense our optical systems are realized in geometry.

It is in general quite hard to derive $\epsilon(\omega), \mu(\omega)$ for a certain given material. In this paper we take a somehow different road and we compute $\epsilon(\omega), \mu(\omega)$ for a particular class of strongly coupled plasma coupled to an electromagnetic field. The media are characterized by two main parameters: the temperature and the chemical potential.

We use the holographic principle to study these systems: the strongly coupled phase of a quantum field theory living in four dimensional Minkowski space time can be described in term of classical gravity in a five dimensional Anti de Sitter space $\left(\mathrm{AdS}_{5}\right)$. The temperature, and the chemical potential of the field theory are encoded in the temperature and charge of a black hole solution in $\mathrm{AdS}_{5}$.

In this minimal setup our media are described by an energy momentum tensor $T_{\mu \nu}$ and a conserved $\mathrm{U}(1)$ current $J_{\mu}$. In the holographic prescription they correspond to a five dimensional metric $g_{m n}$ and a five dimensional vector $A_{m}$ respectively. Their dynamics is described by the Maxwell-Einstein action:

$$
S=\frac{1}{2 e^{2} l^{2}} \int d^{5} x \sqrt{-g}\left(R+\frac{6}{l^{2}}\right)-\frac{1}{4 e^{2}} \int d^{5} x \sqrt{-g} F_{m n} F^{m n}
$$

where $6 / l^{2}$ is the cosmological constant. The ground state of our systems is described by 
the particular solution of the equation of motions of (4.1):

$$
\begin{aligned}
d s^{2} & =\frac{(2-a)^{2} l}{16 b^{2}} \frac{1}{u}\left(\mathrm{~d} x^{2}+\mathrm{d} y^{2}+\mathrm{d} z^{2}-f(u) \mathrm{d} t^{2}\right)+\frac{l^{2}}{4} \frac{\mathrm{d} u^{2}}{u^{2} f(u)} \\
A_{t} & =-\frac{u}{2 b} \sqrt{\frac{3}{2} a}+\Sigma
\end{aligned}
$$

where $\Sigma$ is the chemical potential and

$$
f(u)=(1-u)\left(1+u-a u^{2}\right)
$$

The solution (4.2) is a charged black hole in $\mathrm{AdS}_{5}$ : the event horizon is at $u=1$, while the AdS boundary is at $u=0$. The chemical potential and the temperature of the system are encoded into the parameter $a$ and $b$ through the relations

$$
T=\frac{2-a}{4 \pi b} \quad \Sigma=\frac{1}{2 b} \sqrt{\frac{3}{2} a}
$$

The propagation of the five dimensional photon $A_{m}$ defines the retarded current-current green function for our $(3+1)$ d media. It is necessary to compute $G_{T}^{(0)}(\omega)$ and $G_{T}^{(2)}(\omega)$ to obtain $\epsilon(\omega)$ and $\mu(\omega)$ defined in the previous section.

The procedure to compute these functions using holography is well-established [52]. To compute the $(3+1) \mathrm{d}$ transverse retarded green function $G_{T}(w, k)$ we need to linearize around the solution (4.2) the equations of motions of (4.1) for the components of the $5 \mathrm{~d}$ photon $A_{m}$ transverse to the wave vector k. The equations for the photon are coupled to the equations for the metric and they decoupled only in the limit of zero charge or zero wave vector $(a \rightarrow 0$ or $k \rightarrow 0)$. We then need to solve these equations imposing the infalling boundary condition at the horizon $u=1$. This boundary condition correspond to the physical requirement that nothing can escape from the $5 \mathrm{~d}$ black hole at classical level, and it is the retarded prescription for correlators of the $(3+1) \mathrm{d}$ medium. From the solution for the five dimensional photon we obtain the retarded current-current correlator for the media and hence the linear response function $\epsilon(\omega)$ and $\mu(\omega)$.

This system of equation was solved numerically in [53] and analytically in the hydrodynamic limit in [54-56]. ${ }^{5,6}$ The hydrodynamic approximation is a series expansion in $\omega$ and $k$ for the equations and solutions. It is valid only for small values of $\omega$ and $k$, but it offers analytic expressions. In appendix B we give the explicit form of the retarded correlator at the lowest order in $\omega$ and $k^{2}$. Its leading behavior, necessary for the calculation of $\epsilon(\omega)$ and $\mu(\omega)$, is given by

$$
G^{(0)}(\omega)+k^{2} G^{(2)}(\omega)=\frac{l}{4 b(1+a) e^{2}}\left(\frac{3 a}{b}-i \omega \frac{(2-a)^{2}}{2(1+a)}\right)-\frac{i}{\omega} \frac{3 a l}{8(1+a)^{2} e^{2} b} k^{2}
$$

\footnotetext{
${ }^{5}$ See also [57] in which the authors provided a systematic solution of the wave equations in long wavelength expansion

${ }^{6}$ See also [58] for a previous calculation of these Green functions on $2+1$ dimensions.
} 
Using the equations $(2.10)$ we obtain: ${ }^{7}$

$$
\begin{aligned}
\epsilon(\omega) & =1+q^{2} \frac{l \pi}{b(1+a) e^{2}}\left(\frac{i}{\omega} \frac{(2-a)^{2}}{2(1+a)}-\frac{1}{\omega^{2}} \frac{3 a}{b}\right) \\
\mu(\omega) & \simeq 1+q^{2} \frac{i}{\omega} \frac{3 \pi a l}{2(1+a)^{2} e^{2} b}+\mathcal{O}\left(q^{4}\right)
\end{aligned}
$$

The form of $G_{T}(\omega, k), \epsilon(\omega), \mu(\omega)$, is exactly as in (3.2), (3.3), (3.4), except for an higher order term in $\omega$ that does not influence the conclusion of the previous section. Indeed it is interesting to observe that as soon as we turn on the electric charge of the black hole, i.e. the medium has a non vanishing chemical potential, the low frequency behavior of the real part of $\epsilon(\omega)$ changes discontinuously to negative value, while the imaginary part of $\mu(\omega)$ acquires a positive contribution. Even if $\operatorname{Re}(\mu(\omega))$ never becomes negative, the dissipation and the fact that $\operatorname{Re}(\epsilon(\omega))<0$ conspire to give negative refractive index at low frequencies. ${ }^{8}$ In the next section we will go beyond the hydrodynamic regime and we will compute correlators and response functions for every frequency $\omega$.

\section{$5 \quad$ Numerics and plots}

To go beyond the hydrodynamic limit we need to numerically integrate the equations of motion for the $5 \mathrm{~d}$ gauge field and the metric. The functions $\epsilon(\omega), \mu(\omega)$ are obtained from the correlators $G_{T}^{(0)}(\omega)$ and $G_{T}^{(2)}(\omega)$. We obtain these Green functions expanding the equations in the wave vector $k$ up to the order $k^{2}$ and numerically integrate the resulting equations for every value of $\omega$. As usual the solutions in AdS have IR divergences, that correspond to UV divergences in the dual field theory, that we need to subtract. The holographic renormalization procedure [59] leaves the freedom to add a term to the Green functions of the form $c\left(\omega^{2}-k^{2}\right)$. The constant $c$ represents a renormalization ambiguity that has to be fixed. Our prescription is to require that the electric permittivity approaches 1 at large frequencies. This is a natural physical requirement: for infinitely rapid variation of the fields the medium does not have the time to adapt and it behaves like the vacuum.

With these Green functions we can study the optical properties of the medium that we are describing using gauge/gravity duality and we can check if it shows negative refraction of light, once coupled to a dynamical photon.

Here we plot all the results by fixing $T=1$ for simplicity and by varying the chemical potential from $\Sigma \simeq 3.5$ to $\Sigma \simeq 52$, i.e. $\Sigma>T$.

We first have to ensure that the system is in thermodynamical equilibrium. As we explain in the appendix A, a system in thermodynamical equilibrium must satisfy the

\footnotetext{
${ }^{7}$ We consider a perturbative series expansion in the $(3+1) d$ EM coupling $q$. The validity of the perturbative expansion would imply a lower bound on the frequencies $\omega$, once all the other physical parameters of the systems are fixed.

${ }^{8}$ The negative quadratic pole in $\epsilon(\omega)$ is related at least in part to the translation invariance of the systems we are considering [39-43]. Indeed in translationally invariant systems the conductivity $\sigma(\omega)$ has an imaginary pole $i / \omega$, and $\epsilon(\omega)=1+4 \pi i \sigma(\omega) / \omega$. However we have checked that the refractive index is still negative even if we add a small amount of impurities that break the translation invariance.
} 


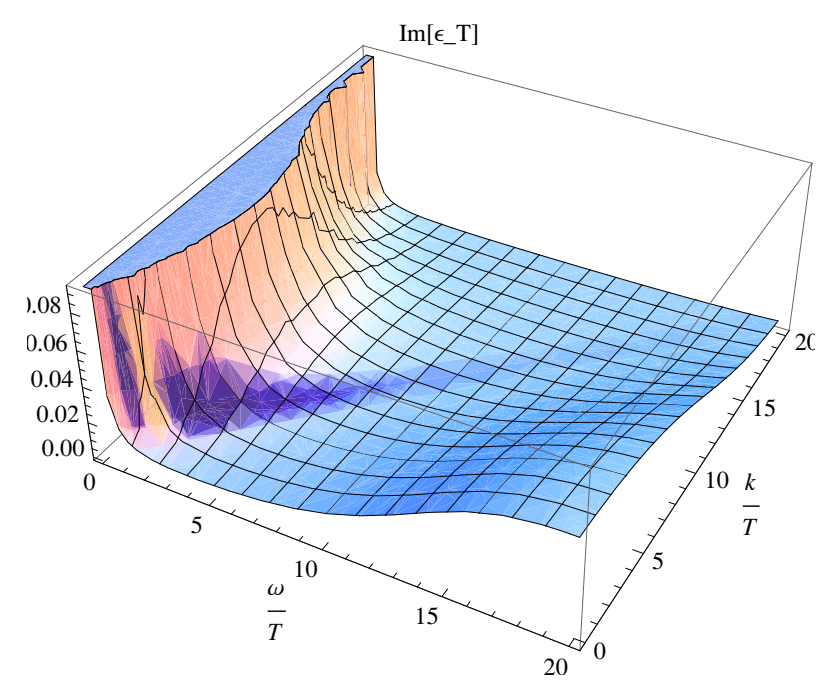

Figure 2. $\operatorname{Im}\left(\epsilon_{T}\right)$ as a function of $\frac{\omega}{T}$ and $\frac{k}{T}$, with $\Sigma / T=7.5$ and $q=0.05$.
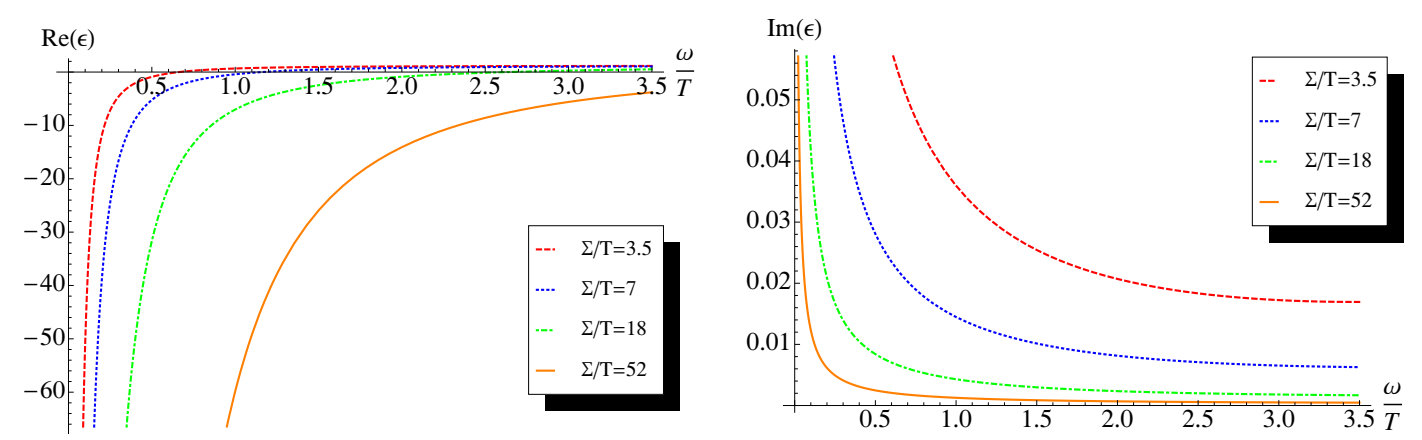

Figure 3. $\operatorname{Re}[\epsilon(\omega)]$ and $\operatorname{Im}[\epsilon(\omega)]$ for different values of $\Sigma / T$ and $q=0.05$.

requirement $\operatorname{Im}\left(\epsilon_{T}\right)>0$, and we expect our system to satisfy this requirements since $\epsilon_{T}$ is given by a Green function. We have checked this property by plotting this function in figure 2 for real $\omega$ and $k$. For this plot we have numerically computed $G_{T}(\omega, k)$ at all orders also in $k$.

Then we plot the real and imaginary parts of the electric permittivity and of the magnetic permeability, in figure 3 and 4 respectively. Notice that $\operatorname{Re}(\mu(\omega))$ and $\operatorname{Re}(\epsilon(\omega))$ are not simultaneously negative. As we stressed in section 3 , also if $\operatorname{Re}(\epsilon) \cdot \operatorname{Re}(\mu)<0$ the energy and phase velocity can be in opposite directions, because of the role played by the imaginary parts, and the material has negative light refraction.

Indeed from figure 1 we see that the index $n_{D L}$ (3.1) is negative at small frequencies for different values of $\Sigma / T$. In the limit $\Sigma / T \rightarrow 0$ we reduce to the uncharged case, and the index is positive also at small frequencies. For small charge $\Sigma / T$, the index becomes immediately negative at small frequencies, and then it become positive for larger $\omega$. By increasing the value of $\Sigma / T$ the region of negative refraction grows. 

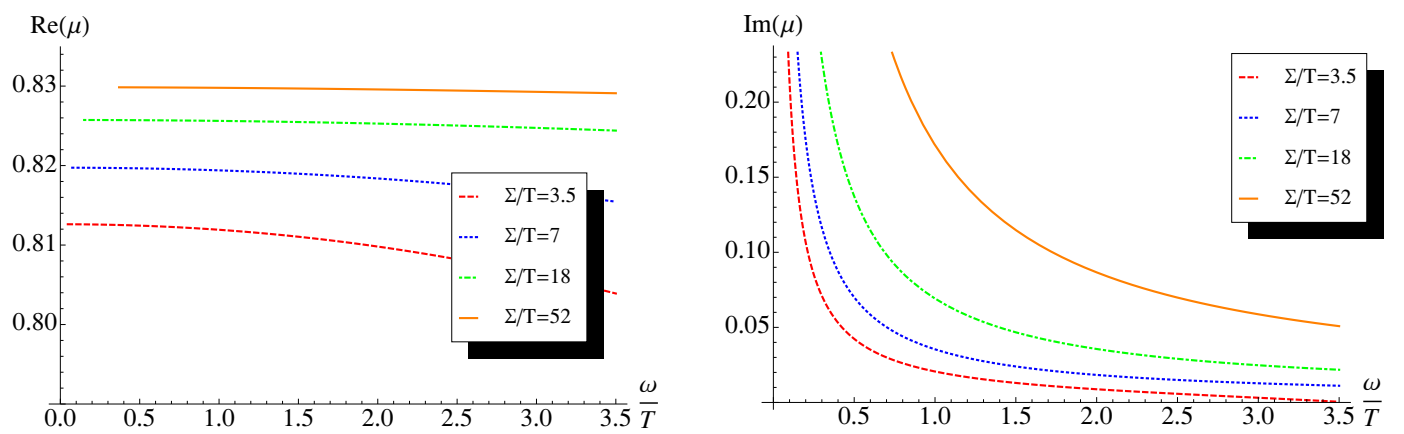

Figure 4. $\operatorname{Re}[\mu(\omega)]$ and $\operatorname{Im}[\mu(\omega)]$ for different values of $\Sigma / T$ and $q=0.05$.

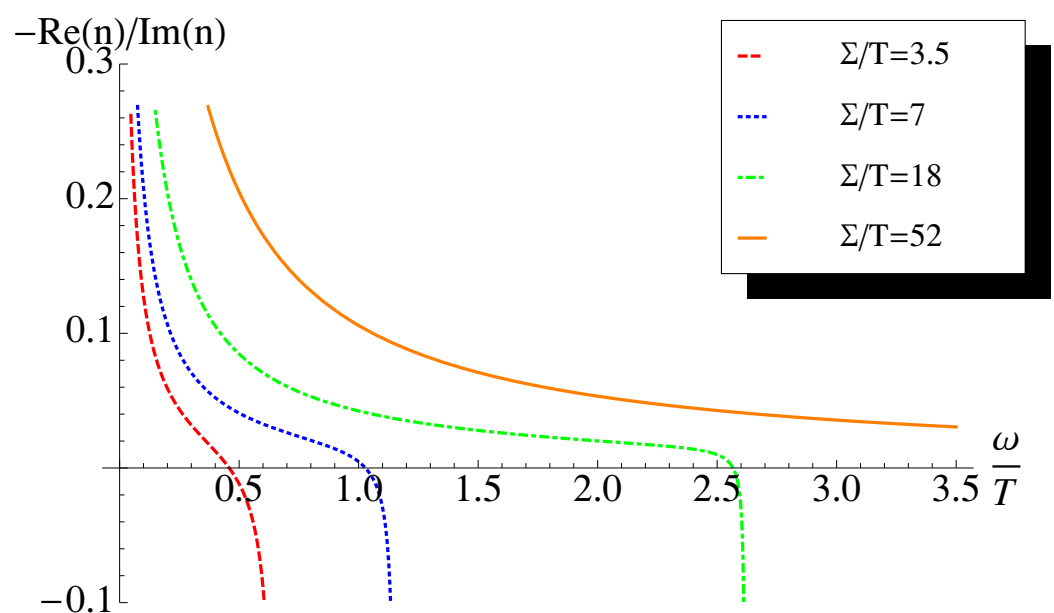

Figure 5. Ratio $-\operatorname{Re}[n(\omega)] / \operatorname{Im}[n(\omega)]$ for different values of $\Sigma / T$ and $q=0.05$.

A last useful quantity to plot is the ratio $\operatorname{Re}(n) / \operatorname{Im}(n)$, in figure 5. Indeed this quantity takes into account the ratio between propagation and dissipation. Usually in isotropic metamaterials it is common to have strong dissipation in the regime of negative refraction [60-63]. This implies that $\left|\frac{\operatorname{Re}(n)}{\operatorname{Im}(n)}\right| \ll 1$. Here we see that this is the case too. The situation improves as $\omega \rightarrow 0$, where the effects of dissipation get lower. At larger frequencies instead the dissipation dominates over the propagation.

As an aside remark, we have found that at higher frequencies $\operatorname{Im}(\mu(\omega))$ becomes negative. We stress that it is not a signal of any instability, since $\epsilon_{T}(\omega, k)$ is always a well defined response function for every value of $\omega$, in particular it always has positive imaginary part. We could interpret this phenomenon as the breakdown of the $\epsilon-\mu$ approach; it would be desirable to have a better understanding of this point. 


\section{Conclusions and future directions}

In the last decade there was a revolution in the practical ability to control the electromagnetic fields in various media and the possibility to study exotic optical phenomena. In this scenario negative refraction of light has been shown to be possible in artificial metamaterials. One can wonder if there are other physical systems with this property. In this paper we concentrated on relativistic field theories at finite temperature and chemical potential.

We guessed that negative refraction is generic in the hydrodynamic limit of these theories. This behavior is based on the form of the retarded propagator of the transverse current. We confirmed this observation with an explicit example. Indeed we studied the optical properties of some strongly coupled media from the perspective of the gauge/gravity correspondence. In this case there is a procedure to exactly compute the transport coefficients and the response functions in terms of the temperature and the chemical potential, and we can obtain quantitative informations about the refractive index.

We argued that a charged black hole in $\mathrm{AdS}_{5}$ is a good laboratory for the study of this unusual optical property of three dimensional media. In this background we have found the range of frequency at which the index of refraction is negative. Even if we studied a specific background, our model is rather generic. Indeed the holographic description presented in section 4 is valid for every strongly coupled media with at least a U(1) global symmetry. It is important to stress that we are not studying the optical properties of the complicate five dimensional curved background. This background is only necessary to compute the correlators of the dual strongly coupled $3+1$ dimensional field theory, whose geometry is flat. Otherwise non-flat geometries lead to other non-conventional optics phenomena (see for example [64]).

There are many possible future directions of research.

On a general ground it would be interesting to find if other amazing phenomena coming from the recent optics developments can be usefully realized in string theory, or if the ability of string theory to describe some peculiar state of matter could help in optic devices.

The U(1) gauge symmetry in the five dimensional gravity background is typically understood as a global symmetry in the dual $3+1$ dimensional field theory. We hope that the possibility of negative refraction could stimulate the efforts towards a better understanding of the gauging procedure in AdS/CFT [65]. Positive results in this direction could give the possibility to actually compute the first quantum electro-dynamical corrections to the refractive index.

As a matter of fact we noted that the dissipation is always stronger than the propagation at frequencies corresponding to negative values of the index of refraction. It is a common property of many isotropic media [60-63], and some ad-hoc engineering procedure have been used to reduce losses in metamaterials. It should be interesting to find an analogy of this procedure in the system that we studied. Maybe one can try to lower the dissipative effects by studying deformations of the holographic background.

The media we studied in this paper are homogeneous and isotropic. To make better contact with real materials it would be interesting to break homogeneity and isotropy 
and to compute current-current correlators in these less symmetric backgrounds [66-68]. Another possibility is the breakdown of the relativistic symmetry [24, 25, 69].

Negative refraction has been observed also in two dimensional materials [70, 71], and it seems natural to extend our analysis of the optical properties from three dimensional media to two dimensional ones at weak coupling and at strong coupling (by using the $\mathrm{AdS}_{4} / \mathrm{CFT}_{3}$ correspondence).

Another natural extension is the study of optical properties of material in the superconducting phase. Indeed negative refraction has been discussed in superconductors in [72, 73], and maybe the same property can be studied in holographic superconductors [19-23] (see also [39-43] and reference therein).

We conclude by noticing the analogy between negative refraction in photonics crystals and the motion of the electrons in a bipolar junction in graphene [74]. It can be interesting to better understand this analogy and we hope that our work can shed new light on the holographic dual of this effect.

\section{Acknowledgments}

It is a great pleasure to thank V. M. Agranovich, C. Bachas, F. Chevy, B. Doucot, Y. Gartstein, V. Ginis, S. Hartnoll, A. Lakhtakia, K. Jo, L. Mazzanti, Y. Oz, A. Salvio, A. Sen, A. Starinets, P. Thassin, J. Troost, I. Veretennicoff and A. Zaffaroni for nice discussions and clarifications. A.A. is supported by UCSD grant DOE-FG03-97ER40546; A. M. is a Postdoctoral researcher of FWO-Vlaanderen. A. M. is also supported in part by the Belgian Federal Science Policy Office through the Interuniversity Attraction Pole IAP VI/11 and by FWO-Vlaanderen through project G.0114.10N; D. F. and G. P. are supported by CNRS and ENS Paris. A. A., D. F. and A. M. thanks the organizers of the Avogadro Meeting and of the INFN Vietri conference and the University of Milano-Bicocca where part of this work has been done. G. P. thanks the NORDITA institute where part of this work was completed.

\section{A Wave propagation and dissipation}

In this appendix we derive the conditions for negative refraction and the consistency conditions for the $\epsilon(\omega, k)$ imposed by thermodynamics.

All along this paper we use the single function $\epsilon(\omega, k)$ to take into account all the interactions between the medium and the electromagnetic field, the charges and the currents. In this setup the Maxwell equations take the following form:

$$
\nabla \cdot D=0, \quad \nabla \cdot B=0, \quad \nabla \wedge E=-\partial_{t} B, \quad \nabla \wedge B=\partial_{t} D
$$

completed by the constitutive relation, written in the dual Fourier space, $D=\epsilon E$. The propagation/dissipation of the electromagnetic field in a dispersive/dissipative medium is a bit tricky.

To determine the propagation of energy in the medium, i.e. the Poynting vector, we use the approach to consider the field coupled to an external source that compensates 
the energy losses due to the dissipation. In such a way we can think of the medium as almost transparent, and the $\epsilon$ is a real function of two real coordinates: $\omega, k$. Analyzing the propagation of a wave packet we obtain the well-known expression for the Poynting vector in the transparency regime for a dispersive medium [44]. To take the dissipation of energy into account, we just need to analytically continue the expression to complex values for $k$ and $\epsilon$. The final expression for the Poynting vector is:

$$
\mathbf{S}=\operatorname{Re}\left(\mathbf{E}^{*} \wedge \mathbf{B}-\frac{\omega}{2} \frac{\partial \epsilon_{i j}}{\partial \mathbf{k}} E_{i}^{*} E_{j}\right) .
$$

The Poynting vector is the energy flow. For transverse waves (A.2) reduces to:

$$
\mathbf{S}=\operatorname{Re}\left(\frac{\mathbf{k}}{\omega}-\frac{\omega}{2} \frac{\partial \epsilon_{T}}{\partial \mathbf{k}}\right)\left|E_{T}\right|^{2}
$$

If we expand for small values of $k$ as in (2.5) we obtain the usual expression for the Poynting vector in a dissipative medium in the $\epsilon-\mu$ formalism:

$$
\mathbf{S}=\operatorname{Re}\left(\frac{n}{\mu}\right)\left|E_{T}\right|^{2} .
$$

where we have used the relation $k / \omega=n$. There is negative refraction when the phase velocity is opposed to the flux of energy:

$$
\operatorname{Re}(n)<0, \quad \operatorname{Re}\left(\frac{n}{\mu}\right)>0
$$

Working with these relations, one can show that (A.5) is equivalent to $n_{D L}(\omega)<0$. This is the condition we use in the main text to check if the refractive index is negative, which was derived in [47].

Let us now consider the constraints imposed by the thermodynamics on the $\epsilon(\omega, k)$. The divergence of $S$ gives the rate of change of the local electromagnetic energy in a medium. To determine the dissipation we consider a monochromatic wave and we take the divergence of (A.2). The first term of (A.2) gives $\operatorname{Re}\left(E^{*} \partial_{t} D+B^{*} B\right)$, while the divergence of the second term is zero for a plane wave. The time average of minus the divergence of the Poynting vector is the energy inflow from the external source needed to maintain the plane wave in the medium. Because in a dissipative medium all the energy of the wave will be eventually transformed in heat flow, this quantity is equivalent to the outgoing heat flow; for transverse waves one has:

$$
Q=\operatorname{Im}\left(\epsilon_{T}\right)\left|E_{T}\right|^{2}
$$

Our system, in absence of the external electromagnetic wave perturbation, is in thermal equilibrium. Hence the energy flow must be positive: $Q>0$. We obtain the consistency condition: $\operatorname{Im}\left(\epsilon_{T}(\omega, k)\right)>0$. Our epsilon functions are derived from retarded Green functions in a causal, unitary field theory for which $\operatorname{Im}(G(\omega, k))<0$. For this reason the consistency condition for the epsilon is automatically satisfied, ${ }^{9}$ as shown in figure 2 .

\footnotetext{
${ }^{9}$ The inequality holds for $k$ real and $\omega$ lying in the upper complex half-plane; again this condition is off-shell from the point of view of a photon propagating in the medium but we imagine to work with an external field that maintains the electromagnetic wave.
} 


\section{B Finding the correlators}

In this appendix we describe the calculation of the retarded Green functions that we used in the paper [53-56]. We need to solve the equations of motion for the fluctuations of the metric and the Maxwell field around the solution (4.2). However these equations are coupled and it is better to define the new variables

$$
\Phi_{ \pm}(u)=\frac{1}{u} h_{t}^{x \prime}(u)-3 a B_{x}(u)+\frac{C_{ \pm}}{u} B_{x}(u) \quad\left(\text { with } C_{ \pm}=(1+a) \pm \sqrt{(1+a)^{2}+3 a b^{2} k^{2}}\right)
$$

$h_{t}^{x}(u)$ is a metric fluctuation and $B_{x}(u)=\frac{A_{x}(u)}{\Sigma}$, with $A_{x}(u)$ a gauge field fluctuation. The equation of motions for the master variables $\Phi_{ \pm}$are

$$
\Phi_{ \pm}^{\prime \prime}(u)+\frac{\left(u^{2} f(u)\right)^{\prime}}{u^{2} f(u)} \Phi_{ \pm}^{\prime}(u)+\frac{b^{2}}{u f^{2}(u)}\left(\omega^{2}-k^{2} f(u)\right) \Phi_{ \pm}(u)-\frac{C_{ \pm}}{f(u)} \Phi_{ \pm}(u)=0
$$

It is necessary to solve these equations and find the expression for $B_{x}^{\prime}(u)$ to obtain the lorentzian correlators.

The analytical solution is found at the lowest order in $\omega$ and $k^{2}$. First we must impose the infalling boundary condition at the horizon $u=1$. This constrains the solution to be of the form $\Phi_{ \pm}(u)=(1-u)^{-\frac{i \omega b}{(2-a)}} \phi_{ \pm}$. The functions $\phi_{ \pm}$are found by solving the equations at the lowest order. After imposing the boundary conditions for all the fields at $u=0$, the derivative of $B_{x}(u)$ at the boundary is given by

$$
B_{x}^{\prime}(u)=-\frac{\left(\omega k b\left(h_{z}^{x}\right)^{0}+k^{2} b\left(h_{t}^{x}\right)^{0}\right)+3 i a \omega B_{0}}{2 i \omega(1+a)-b k^{2}}+i \frac{(2-a)^{2} b \omega B_{0}}{4(1+a)^{2}}+b^{2} k^{2} B_{0} \log (u)
$$

The retarded Green function is obtained from the boundary action

$$
S \sim-\frac{3 a l}{32 e^{2} b^{4}} \int \frac{d^{4} k}{(2 \pi)^{4}} f(u) B_{x}(-k, u) B_{x}^{\prime}(k, u)
$$

by using the prescription of [52] for the minkowskian correlator, and subtracting the UV divergencies:

$$
G_{x x}=\frac{i \omega l}{e^{2}}\left(\frac{3 a}{2 b^{2}\left(2 i \omega(1+a)-b k^{2}\right)}-\frac{(2-a)^{2}}{8(1+a)^{2} b}\right)
$$

To obtain a result valid for every value of $\omega$ and $k$, we need to implement the above procedure at numerical level.

Open Access. This article is distributed under the terms of the Creative Commons Attribution Noncommercial License which permits any noncommercial use, distribution, and reproduction in any medium, provided the original author(s) and source are credited.

\section{References}

[1] U. Leonhardt and T.G. Philbin, Transformation Optics and the Geometry of Light, arXiv:0805. 4778. 
[2] D.R. Smith et al., Composite Medium with Simultaneously Negative Permeability and Permittivity, Phys. Rev. Lett. 84 (2000) 4184.

[3] J.B. Pendry, Negative Refraction Makes a Perfect Lens, Phys. Rev. Lett. 85 (2000) 3966.

[4] V. Veselago, L. Braginsky, V. Shklover and C. Hafner, Negative Refractive Index Materials, J. Comput. Theor. Nanosci. 3 (2006) 189.

[5] S.A. Ramakrishna, Physics of negative refractive index materials, Rep. Prog. Phys. 68 (2005) 449.

[6] J. Valentine et al., Three-dimensional optical metamaterial with a negative refractive index, Nature 455 ( 2008) 376.

[7] D.A. Genov, S. Zhang and X. Zhang, Mimicking celestial mechanics in metamaterials, Nat. Phys. 5 (2009) 687.

[8] V.G. Veselago, The electrodynamics of substances with simultaneously negative values of $\epsilon$ and $\mu$, Sov. Phys. Usp. 10 (1968) 509.

[9] V.M. Agranovich and Y.N. Gartstein, Spatial dispersion and negative refraction of light, Phys. Usp. 49 (2006) 1029.

[10] V.M. Agranovich, Y.R. Shen, R.H. Baughman and A.A. Zakhidov, Optical bulk and surface waves with negative refraction, J. Lumin. 110 (2004) 167.

[11] V.M. Agranovich and Y.N. Gartstein, Electrodynamics of metamaterials and the Landau-Lifshitz approach to the magnetic permeability, Metamaterials 3 (2009) 19.

[12] http://www.wave-scattering.com/negative.html.

[13] R.A. Shelby, D.R. Smith and S. Schultz, Experimental Verification of a Negative Index of Refraction, Science 292 (2001) 77.

[14] J.M. Maldacena, The large- $N$ limit of superconformal field theories and supergravity, Int. J. Theor. Phys. 38 (1999) 1113 [Adv. Theor. Math. Phys. 2 (1998) 231] [hep-th/9711200] [SPIRES].

[15] O. Aharony, S.S. Gubser, J.M. Maldacena, H. Ooguri and Y. Oz, Large-N field theories, string theory and gravity, Phys. Rept. 323 (2000) 183 [hep-th/9905111] [SPIRES].

[16] G. Policastro, D.T. Son and A.O. Starinets, From AdS/CFT correspondence to hydrodynamics, JHEP 09 (2002) 043 [hep-th/0205052] [SPIRES].

[17] D.T. Son and A.O. Starinets, Viscosity, Black Holes and Quantum Field Theory, Ann. Rev. Nucl. Part. Sci. 57 (2007) 95 [arXiv:0704.0240] [SPIRES].

[18] E. Shuryak, Physics of Strongly coupled quark-gluon Plasma, Prog. Part. Nucl. Phys. 62 (2009) 48 [arXiv:0807.3033] [SPIRES].

[19] S.A. Hartnoll, C.P. Herzog and G.T. Horowitz, Building a Holographic Superconductor, Phys. Rev. Lett. 101 (2008) 031601 [arXiv:0803.3295] [SPIRES].

[20] C.P. Herzog, P.K. Kovtun and D.T. Son, Holographic model of superfluidity, Phys. Rev. D 79 (2009) 066002 [arXiv:0809.4870] [SPIRES].

[21] S.A. Hartnoll, C.P. Herzog and G.T. Horowitz, Holographic Superconductors, JHEP 12 (2008) 015 [arXiv:0810.1563] [SPIRES].

[22] S.S. Gubser, Breaking an Abelian gauge symmetry near a black hole horizon, Phys. Rev. D 78 (2008) 065034 [arXiv:0801.2977] [SPIRES]. 
[23] C.P. Herzog, P.K. Kovtun and D.T. Son, Holographic model of superfluidity, Phys. Rev. D 79 (2009) 066002 [arXiv:0809.4870] [SPIRES].

[24] D.T. Son, Toward an AdS/cold atoms correspondence: a geometric realization of the Schroedinger symmetry, Phys. Rev. D 78 (2008) 046003 [arXiv: 0804.3972] [SPIRES].

[25] K. Balasubramanian and J. McGreevy, Gravity duals for non-relativistic CFTs, Phys. Rev. Lett. 101 (2008) 061601 [arXiv:0804.4053] [SPIRES].

[26] J.L. Davis, P. Kraus and A. Shah, Gravity Dual of a Quantum Hall Plateau Transition, JHEP 11 (2008) 020 [arXiv: 0809.1876] [SPIRES].

[27] M. Fujita, W. Li, S. Ryu and T. Takayanagi, Fractional Quantum Hall Effect via Holography: Chern-Simons, Edge States and Hierarchy, JHEP 06 (2009) 066 [arXiv:0901.0924] [SPIRES].

[28] O. Bergman, N. Jokela, G. Lifschytz and M. Lippert, Quantum Hall Effect in a Holographic Model, JHEP 10 (2010) 063 [arXiv: 1003.4965] [SPIRES].

[29] H. Liu, J. McGreevy and D. Vegh, Non-Fermi liquids from holography, arXiv:0903.2477 [SPIRES].

[30] M. Cubrovic, J. Zaanen and K. Schalm, String Theory, Quantum Phase Transitions and the Emergent Fermi-Liquid, Science 325 (2009) 439 [arXiv:0904.1993] [SPIRES].

[31] T. Faulkner, H. Liu, J. McGreevy and D. Vegh, Emergent quantum criticality, Fermi surfaces and AdS2, arXiv:0907.2694 [SPIRES].

[32] S.A. Hartnoll, J. Polchinski, E. Silverstein and D. Tong, Towards strange metallic holography, JHEP 04 (2010) 120 [arXiv:0912.1061] [SPIRES].

[33] T. Faulkner, N. Iqbal, H. Liu, J. McGreevy and D. Vegh, From black holes to strange metals, arXiv: 1003.1728 [SPIRES].

[34] N. Evans, A. Gebauer, K.-Y. Kim and M. Magou, Holographic Description of the Phase Diagram of a Chiral Symmetry Breaking Gauge Theory, JHEP 03 (2010) 132 [arXiv: 1002.1885] [SPIRES].

[35] K. Jensen, A. Karch and E.G. Thompson, A Holographic Quantum Critical Point at Finite Magnetic Field and Finite Density, JHEP 05 (2010) 015 [arXiv: 1002.2447] [SPIRES].

[36] K. Jensen, A. Karch, D.T. Son and E.G. Thompson, Holographic Berezinskii-Kosterlitz-Thouless Transitions, Phys. Rev. Lett. 105 (2010) 041601 [arXiv: 1002.3159] [SPIRES].

[37] N. Iqbal, H. Liu, M. Mezei and Q. Si, Quantum phase transitions in holographic models of magnetism and superconductors, Phys. Rev. D 82 (2010) 045002 [arXiv:1003.0010] [SPIRES].

[38] E. D'Hoker and P. Kraus, Holographic Metamagnetism, Quantum Criticality and Crossover Behavior, JHEP 05 (2010) 083 [arXiv: 1003.1302] [SPIRES].

[39] S.A. Hartnoll, Lectures on holographic methods for condensed matter physics, Class. Quant. Grav. 26 (2009) 224002 [arXiv:0903.3246] [SPIRES].

[40] C.P. Herzog, Lectures on Holographic Superfluidity and Superconductivity, J. Phys. A 42 (2009) 343001 [arXiv:0904.1975] [SPIRES].

[41] G.T. Horowitz, Introduction to Holographic Superconductors, arXiv:1002.1722 [SPIRES]. 
[42] J. McGreevy, Holographic duality with a view toward many-body physics, Adv. High Energy Phys. 2010 (2010) 723105 [arXiv:0909.0518] [SPIRES].

[43] S. Sachdev, Condensed matter and AdS/CFT, arXiv:1002.2947 [SPIRES].

[44] L.D. Landau and E.M. Lifshitz, Electrodynamics of continous media, Pergamon Press, Oxford U.K. (1984).

[45] M. Dressel and G. Gruner, Electrodynamics of Solids, Cambridge University Press, Cambridge U.K. (2002).

[46] M.W. McCall, A. Lakhtakia and W.S. Weiglhofer, The negative index of refraction demystified, Eur. J. Phys. 23353 [physics/0204067].

[47] R.A. Depine and A. Lakhtakia, A new condition to identify isotropic dielectric-magnetic materials displaying negative phase velocity, Microwave Opt. Technol. Lett. 41 (2004) 315.

[48] L.P. Kadanoff and P.C. Martin, Hydrodynamic Equations and Correlation Functions, Ann. Phys. 24 (1963) 419.

[49] S.A. Hartnoll, P.K. Kovtun, M. Muller and S. Sachdev, Theory of the Nernst effect near quantum phase transitions in condensed matter and in dyonic black holes, Phys. Rev. B 76 (2007) 144502 [arXiv:0706.3215] [SPIRES].

[50] L.D. Landau, E.M. Lifshitz, Statistical Physics. Part 2, Pergamon Press, Oxford U.K. (1984).

[51] S. Caron-Huot, P. Kovtun, G.D. Moore, A. Starinets and L.G. Yaffe, Photon and dilepton production in supersymmetric Yang-Mills plasma, JHEP 12 (2006) 015 [hep-th/0607237] [SPIRES].

[52] D.T. Son and A.O. Starinets, Minkowski-space correlators in AdS/CFT correspondence: Recipe and applications, JHEP 09 (2002) 042 [hep-th/0205051] [SPIRES].

[53] K. Jo and S.-J. Sin, Photo-emission rate of sQGP at finite density, Phys. Rev. D 83 (2011) 026004 [arXiv:1005.0200] [SPIRES].

[54] X.-H. Ge, Y. Matsuo, F.-W. Shu, S.-J. Sin and T. Tsukioka, Density Dependence of Transport Coefficients from Holographic Hydrodynamics, Prog. Theor. Phys. 120 (2008) 833 [arXiv:0806.4460] [SPIRES].

[55] Y. Matsuo, S.-J. Sin, S. Takeuchi, T. Tsukioka and C.-M. Yoo, Sound Modes in Holographic Hydrodynamics for Charged AdS Black Hole, Nucl. Phys. B 820 (2009) 593 [arXiv:0901.0610] [SPIRES].

[56] Y. Matsuo, S.-J. Sin, S. Takeuchi and T. Tsukioka, Magnetic conductivity and Chern-Simons Term in Holographic Hydrodynamics of Charged AdS Black Hole, JHEP 04 (2010) 071 [arXiv:0910.3722] [SPIRES].

[57] B. Sahoo and H.-U. Yee, Holographic chiral shear waves from anomaly, Phys. Lett. B 689 (2010) 206 [arXiv:0910.5915] [SPIRES].

[58] S.A. Hartnoll and C.P. Herzog, Ohm's Law at strong coupling: $S$ duality and the cyclotron resonance, Phys. Rev. D 76 (2007) 106012 [arXiv:0706.3228] [SPIRES].

[59] K. Skenderis, Lecture notes on holographic renormalization, Class. Quant. Grav. 19 (2002) 5849 [hep-th/0209067] [SPIRES].

[60] M.I. Stockman, Criterion for negative refraction with low optical losses from a fundamental principal of causality, Phys. Rev. Lett. 98 (2007) 177404. 
[61] R. Wua and D. Zou, Phase diagram of lossy negative index metamaterials, Appl. Phys. Lett. 93 (2008) 101106.

[62] J. Zhou, T. Koschny and C.M. Soukoulis, An efficient way to reduce losses of left-handed metamaterials, Optics Express 16 (2008) 11147 [arXiv:0807.2302].

[63] A.J. Mackay, Dispersion requirements of low-loss negative refractive index materials and their realisability, IET Microwaves Antennas Propag. 3 (2009) 808.

[64] T.J. Hollowood, G.M. Shore and R.J. Stanley, The Refractive Index of Curved Spacetime II: QED, Penrose Limits and Black Holes, JHEP 08 (2009) 089 [arXiv:0905.0771] [SPIRES].

[65] O. Domenech, M. Montull, A. Pomarol, A. Salvio and P.J. Silva, Emergent Gauge Fields in Holographic Superconductors, JHEP 08 (2010) 033 [arXiv: 1005.1776] [SPIRES].

[66] S.S. Gubser and F.D. Rocha, Peculiar properties of a charged dilatonic black hole in $A d S_{5}$, Phys. Rev. D 81 (2010) 046001 [arXiv:0911.2898] [SPIRES].

[67] K. Goldstein, S. Kachru, S. Prakash and S.P. Trivedi, Holography of Charged Dilaton Black Holes, JHEP 08 (2010) 078 [arXiv:0911.3586] [SPIRES].

[68] E. Perlmutter, Domain Wall Holography for Finite Temperature Scaling Solutions, JHEP 02 (2011) 013 [arXiv: 1006.2124] [SPIRES].

[69] Y. Nishida and D.T. Son, Nonrelativistic conformal field theories, Phys. Rev. D 76 (2007) 086004 [arXiv:0706. 3746] [SPIRES].

[70] R.A. Shelby, D.R. Smith, S.C. Nemat-Nasser and S. Schultz, Microwave transmission through a two-dimensional, isotropic, left-handed metamaterial, Appl. Phys. Lett. 78 (2001) 489.

[71] K. Aydin, K. Guven and E. Ozbay, Two-dimensional Left-handed Metamaterial with a Negative Refractive Index, J. Phys. Conf. Ser. 36 (2006) 6.

[72] A. Pimenov, A. Loidl, P. Przyslupski and B. Dabrowski, Negative Refraction in Ferromagnet-Superconductor Superlattices, Phys. Rev. Lett. 95 (2005) 247009 [cond-mat/0510777].

[73] A.L. Rakhmanov, V.A. Yampol'skii, J.A. Fan, F. Capasso and F. Nori, Layered superconductors as negative-refractive-index metamaterials, Phys. Rev. B 81 (2010) 075101 [arXiv: 0907.3564].

[74] V.V. Cheianov, V. Fal'ko and B.L. Altshuler, The Focusing of Electron Flow and a Veselago Lens in Graphene p-n Junctions, Science 315 (2007) 1252. 